\title{
LES GENITALIA FEMELLES DES TABANIDES ET LEUR IMPORTANCE EN SYSTÉMATIQUE
}

\author{
Par M. OVAZZA et R. TAUFFLIEB
}

Parmi les Tabanides, les Chrysops du sous-genre Kleineana jouent un ròle de premier plan dans la transmission des affections humaines, puisqu'ils sont les vecteurs de la filariose à Loa loa. D'autres Tabanides, en outre, tiennent une place importante dans l'épidémiologie des trypanosomiases du gros bétail en Afrique. Aussi l'identification de ces Diptères prend-elle de plus en plus d'importance.

S’il est, en général, aisé de différencier les diverses espèces de Chrysops il n'en est pas de même en ce qui concerne les espèces appartenant aux autres genres, surtout quand les spécimens sont secs. La morphologie des genitalia a fort peu été utilisée pour la classification des Tabanides, car les mâles sont difficiles à trouver, particulièrement dans la région éthiopienne. En ce qui concerne les femelles, la plupart des auteurs considèrent leurs pièces génitales comme inutilisables en systématique: Brygoo (1950), après avoir disséqué un certain nombre d'espèces, n’accorde aucune valeur spécifique à ces structures. J. E. Collin (1940), qui a étudié les femelles de trois Therioplectes de Grande-Bretagne, considère comme probablement utilisables les cerques et la plaque ventrale. H. Oldroyd (1952) pense que certains caractères, comme la forme des spermathèques, sont des caractères de groupe, mais que d'autres particularités peuvent, dans certains cas, aider à l'identification.

Nous avons disséqué les femelles de 25 espèces de Tabanides de l'Afrique Equatoriale française. Ces espèces appartiennent aux genres : Tabanus, Haematopota, Hippocentrum, Tabanocella et Chrysops. Nous avons pu, en outre, disséquer un Dorcolaemus qu'a bien voulu nous confier $\mathrm{M}$. le $\mathrm{P}^{\mathrm{r}}$ Roubaud; enfin, à Londres, le $D^{r}$ Oldroyd nous a permis d'étudier deux exemplaires de Tabanus et un Thaumastocera de la collection du British Museum (*). Dans

(*) Ce travail, commencé à l'Institut Pasteur de Brazzaville et à l'Institut d'Etudes Centrafricaines, a été achevé à l'Institut Pasteur de Paris, Service de M. le Prof. Roubaud, Laboratoire de M. le $\mathrm{D}^{\mathrm{r}}$ Grenier.

ANN, de Parastorogie, r. XXIX, N $3 .-1954$. 
l'état actuel de nos connaissances, il nous semble que les senitalia $\odot$ fournissent des caractères utiles pour l'identification. Nous avons d'autre part l'impression qu'un de ces caractères (la forme des spermathèques) permet de séparer les deux sous-familles: Tabaniinæ et Pangoniinæ.

Dans ce premier travail nous donnerons, dans une première partie, une étude de la morphologie de ces pièces (historique des connaissances, discussion, essai d'interprétation) et une rapide description des caractères qui nous ont paru utilisables, en soulignant les différences existant entre les deux sous-familles. La deuxiême partie comprendra la description des genitalia ${ }^{\circ}$ de quatre espèces appartenant à la sous-famille des Pangoniinæe (Tabanocella stimulans Aust. et trois Chrysops du sous-genre Kleineana: Chr. silacea Aust., Chr. dimidiata V. der W., Chr. longicornis Macq.). Dans les articles ultérieurs, nous décrirons les autres espèces que nous avons pu étudier.

\section{f. - Morphologie. Les caractères utilisables.}

\section{A) Historique :}

Patton et Cragg (1913) considèrent que chez la femelle les segments terminaux télescopés forment une sorte d'ovipositeur. Celuici serait composé : d'une plaque dorsale, équivalant au tergite VIII, deux petites plaques latérales représentant les tergites IX et $\mathrm{X}$ et séparés en deux moitiés latérales, deux plaques anales leur faisant suite et se projetant à l'extrémité de l'abdomen (ces plaques anales représenteraient le tergite XI). La paroi ventrale est formée d'une seule plaque médiane représentant, peut-être, les sternites correspondants. Pour ces auteurs, le plus grand fractionnement de la paroi dorsale permettrait à l'ovipositeur de se courber vers le bas lors du dépôt des œufs. Plus loin, à propos des organes génitaux internes, Patton et Cragg signalent (p. 137) : «Sur la poche copulatrice... (existe) un support chiniteux portant une série de petites

Nous tenons à remercier : M. le Médecin-Colonel Ceccaldi, Directeur de l'I.P. de Brazzaville, et M. le Prof. Trochain, Directeur de l'I.E.C., qui nous ont fourni les moyens permettant de faire ce travail; M. le Prof. Roubaud, chez qui nous l'avons achevé, et qui nous a procuré un genre (Dorcoleamus) qui nous manquait; M. le $\mathrm{D}^{\mathrm{r}}$ P. Grenier, qui nous a conseillé, a revu nos dessins et nous a considérablement aidé pour l'interprétation morphologique ; M. le $\mathrm{D}^{\mathrm{r}} \mathrm{H}$. Oldroyd, du British Museum, qui a vérifié nos identifications et a accueilli l'un de nous dans son laboratoire, où il l'a aidé de ses conseils et lui a permis de disséquer un exemplaire de Thaumastocera, genre que nous n'avions pu eapturer. 
épines qui, apparemment, s'engrènent avec les pièces génitales mâles pendant la copulation. » Ils décrivent les spermathèques comme de longs tubes fins, repliés en leur milieu et dont l'extrémité aveugle, légèrement renflée, est attachée à la paroi de l'oviducte près de l'origine du tube. La paroi interne de ce tube est formée d'une chitine qui s'épaissit vers l'extrémité et présente une striation transversale. A la base de chaque tube, là où il se jette dans la poche copulatrice, se trouve un collier chitineux épais en forme de cheminée, entourée d'une série de filaments spiralés comme une trachée.

E. Séguy (1950 et 1951) donne, dans deux publications, des dessins de pièces génitales de Tabanides; il fournit en outre, dans le premier de ces deux travaux, une interprétation morphologique de cet appareil chez les Diptères en général $(1950$, p. 62) : « Chez la femelle l'ovipositeur, tarière ou oviscapte, modifie, comme chez le mâle; les segments VII à X. Le segment X est composé de quatre pièces entourant l'anus : une plaque tergale triangulaire, une plaque sternale arrondie, portant aux angles deux prolongements chitineux et une paire de lamelles terminales (cerques) articulées avec les plaques sternales et tergales. Les cerques correspondent aux gonapophyses de l'appareil génital mâle et protègent l'ouverture anale. 》Dans le dessin correspondant aux Tabanides cet auteur fait figurer une plaque ventrale qu'il nomme «lamelle ventrale unique ».

Pour Collin (1940) la lamelle ventrale de Séguy serait le $8^{\circ}$ sternite.

\section{B) Observations personnelles :}

Nos propres observations nous ont permis de constater l'existence des sclérites suivants (cíf. fig. 1, A et coupes B et C), le $7^{\circ}$ segment est le dernier visible sur un spécimen sec. La dissection montre qu'il existe, invaginé à l'intérieur de celui-ci, un $8^{\circ}$ anneau complet avec sternite (ST VIII) et tergite (T VIII) ; dans l'espace limité par cet anneau sont contenues les pièces suivantes : dorsalement 4 petits sclérites groupés deux par deux autour d'une membrane en croix (c) que nous appelons plaques basales des cerques; elles sont suivies d'une paire de lamelles (c') qui sont les cerques des auteurs. Ventralement, par rapport à cet ensemble, une lame impaire ou lamelle dorsale (f) en forme de triangle ou de losange à angles arrondis ; la dissection la montre reliée à l'ensemble (c) par des membranes latérales et réunie, de la même façon, à un grand 

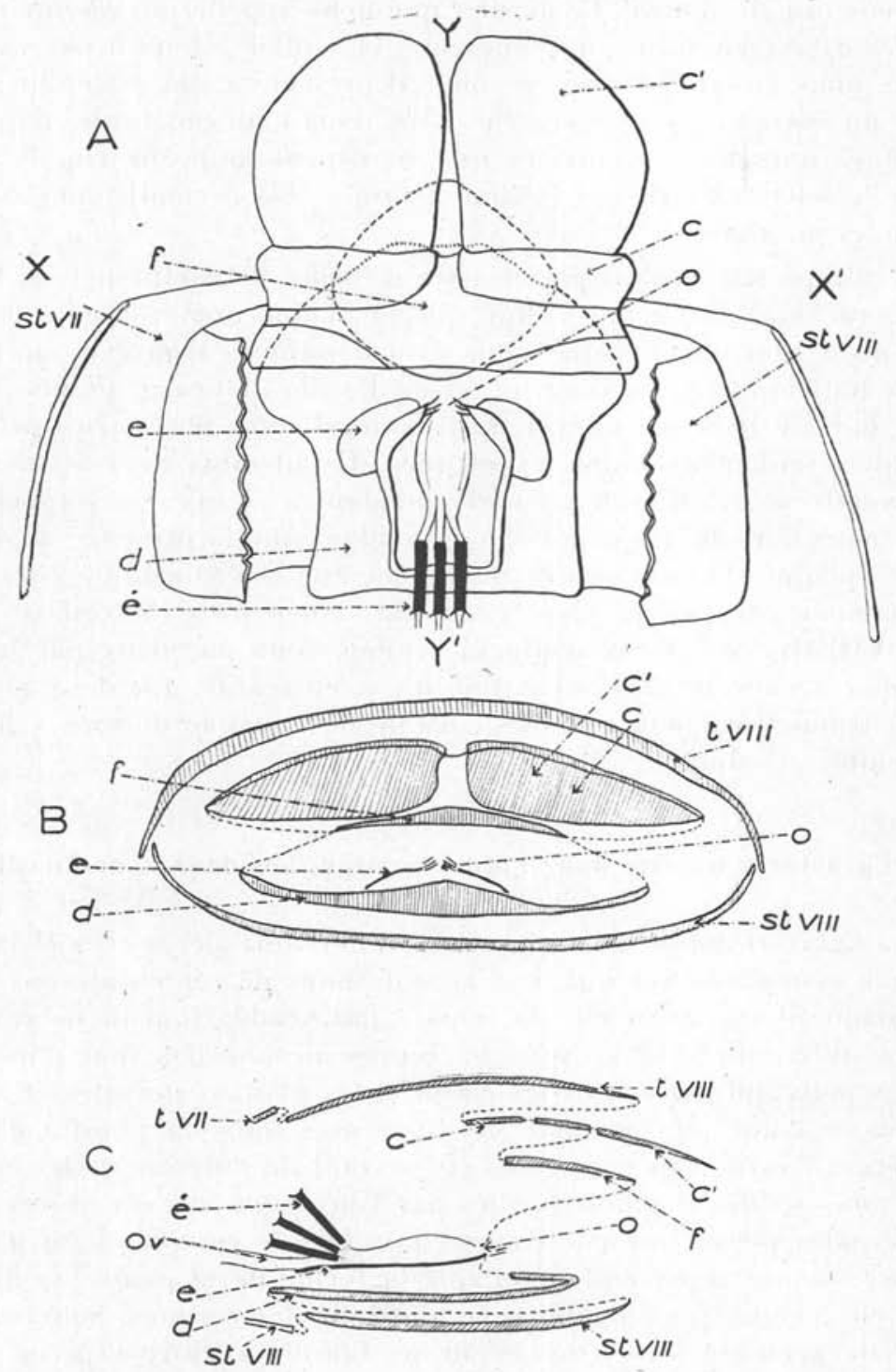

Fig. 1. - Schéma général des genitalia of de Tabanides. A : vue d'ensemble dorsale, tergites VII et VIII enlevés; $\mathrm{B}$ : coupe selon X-X' (sans le septième segment) ; C : coupe selon Y-Y', ST VIII : sternite VIII ; T VIII : tergite VIII; ST VII : sternite VII ; T VII : tergite VII ; $\mathrm{c}$ : pièces basales des cerques ; $c^{\prime}$ : cerques ; $d$ : plaque ventrale ; e : furca ; e' : manchons chitineux des spermathèques; $f$ : plaque dorsale; 0 ; orifice gènital femelle; ov: oviducte. 
sclérite impair ventral. Ce dernier que nous appellerons plaque ventrale (d) est en forme de pentagone irrégulier lorsqu'il est vu en plan, mais lorsqu'il est vu en coupe il présente à son extrémité distale un assez large repli ventral. C'est dans l'anneau formé par ces plaques dorsales et ventrales que se situent la poche copulatrice avec le sclérite décrit par Patton et Cragg, les spermathèques et les glandes annexes.

L'interprétation nous parait assez difficile. Nous éliminerons tout d'abord l'idée émise par Collin que la plaque impaire ventrale (f) est un $8^{\circ}$ sternite, puisque nous avons toujours trouvé ce dernier bien individualisé. La description de Patton et Cragg nomme plaque dorsale le $8^{\circ}$ sternite et mentionne, d'autre part, deux petites plaques seulement avant les cerques. Les dessins de ces auteurs ne montrent pas de sclérite correspondant à ce que nous appelons la plaque dorsale. Il est possible cependant que la présence de quatre « plaques basales des cerques » ne soit pas constante chez les Tabanides. En effet, chez Chrysops longicornis Macquart (ef. fig. VIII, B) ces pièces semblent réunies deux par deux par leurs bords externes, de telle sorte qu'il n'y a, en réalité, que deux pièces symétriques. Par contre, l'existence de la «plaque dorsale » nous a semblé constante.

\section{G) Caractères utilisés pour l'identification. Les deux sous-familles :}

La figure II donne un exemple des différentes pièces chez Haematopota abyssinica Surcouf. Par la suite nous ne représenterons pas la plaque dorsale, car elle ne nous a pas semblé fournir de caractères différentiels sùrs. Nous ne représenterons pas non plus les longs poils qui garnissent les cerques, les plaques dorsales et ventrales, n'ayant pu constater de différences dans la pilosité d'une espèce à l'autre. Les caractères permettant de différencier les espèces nous semblent plutôt formés par l'ensemble des variations des différentes pièces que par l'aspect de l'une de ces pièces en particulier. Il faut cependant noter que la forme de la plaque ventrale est, en général, plus significative que celle des cerques. Souvent ce sclérite présente un certain « air de famille » entre espèces voisines. A cet égard et chez tous les Tabaniinæ que nous avons examinés, il présente une chitinisation plus accentuée latéralement qu'en son milieu ; il est, en outre, marqué d'un certain nombre de stries ou plis transversaux (fig. II, D). Au contraire, chez Tabanocella et les trois Chrysops (fig. V, VI, VII, VIII), sa chitinisation est uniforme et sa surface unie. 

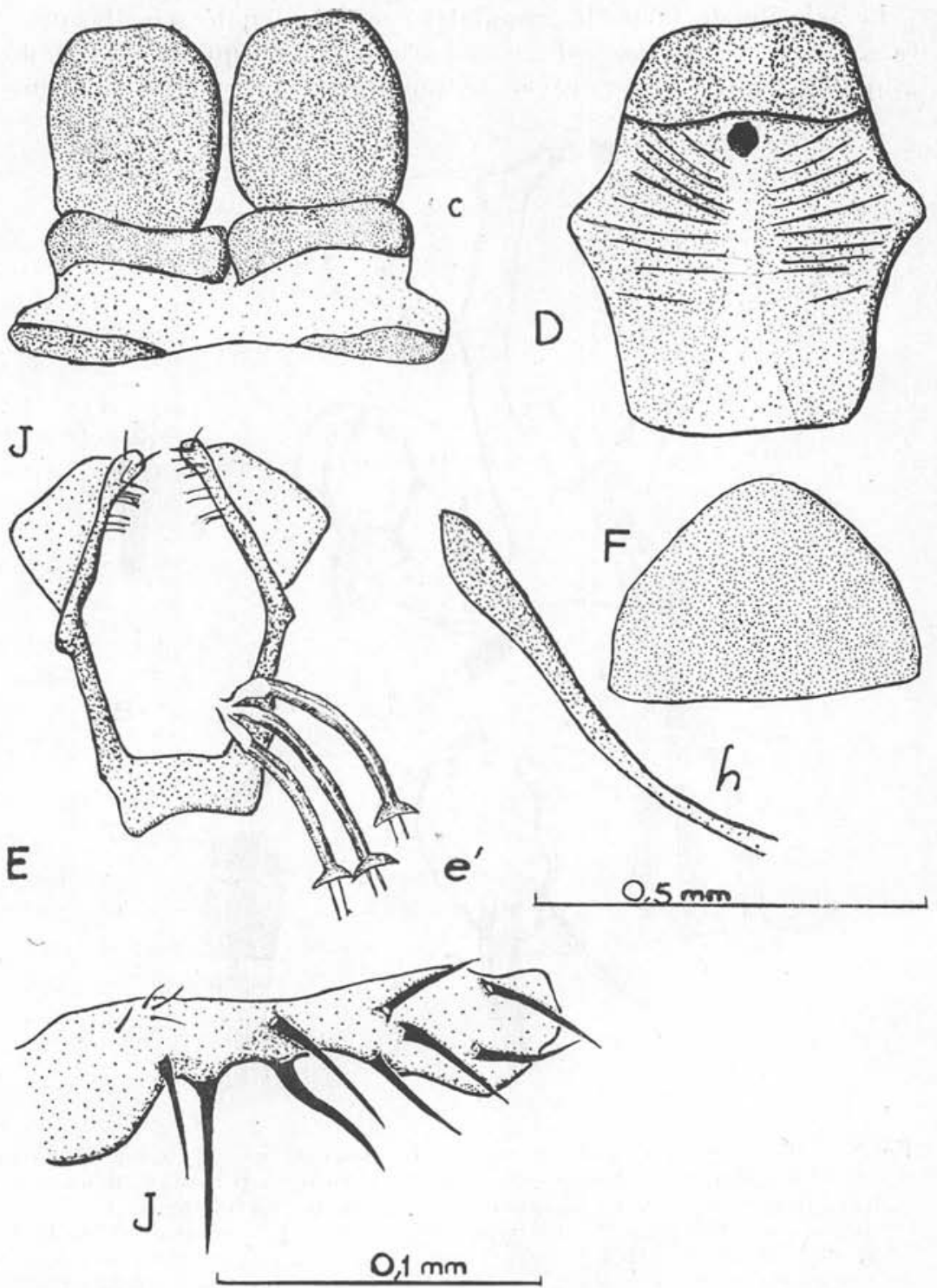

Fic. 2. - Différentes pièces génitales d'un Tabanide $f$ (Hamatopota abyssinica Surcouf), c : cerques et leurs plaques basales; D: plaque ventrale; E : furca ; F : plaque dorsale ; J : extrémité distale d'un bras de la furca avec la « brosse » (e': manchons des canaux des spermathèques : h: une spermathèque). 
Le sclérite de la poche copulatrice a été signalé par Patton et Cragg. J. E. Collin (loc. cit.) n'en parle pas, bien qu'une des photographies jointes à son travail le représente. Le sclérite se trouve

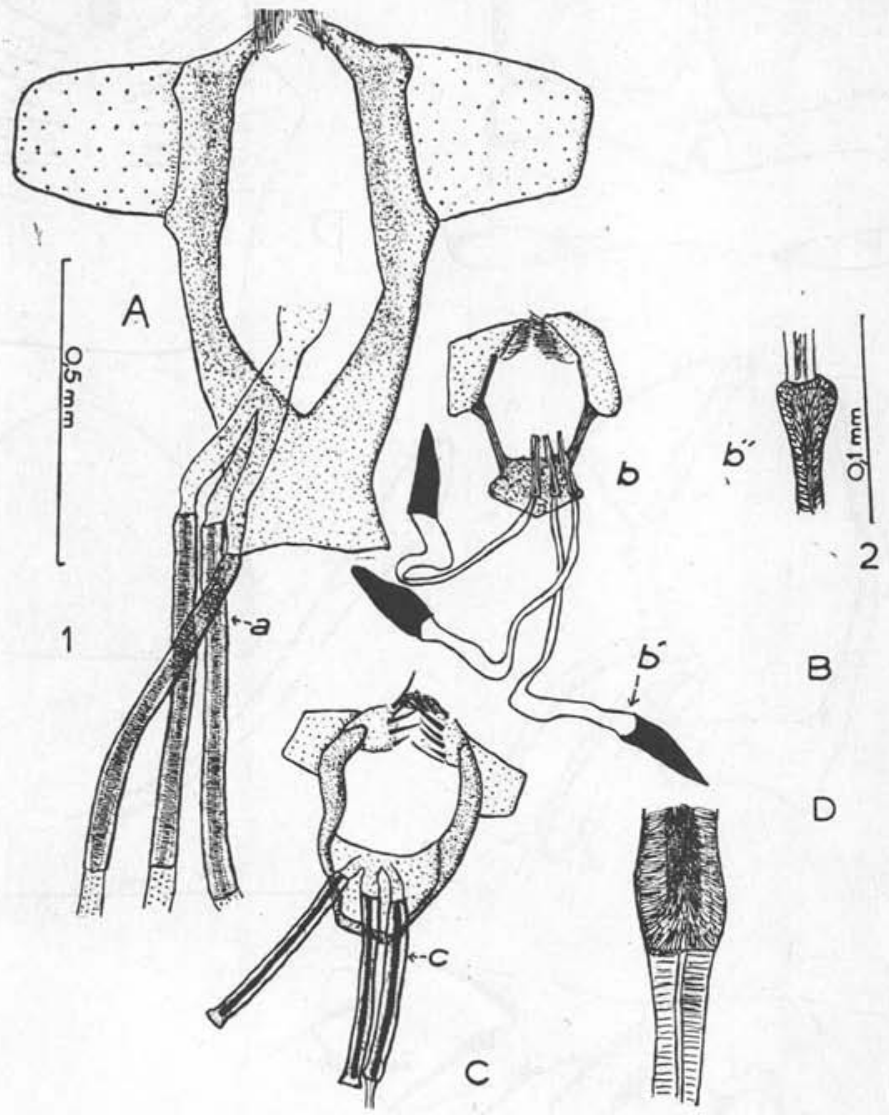

Fid 3. - Furca chez les Pangoniina. A : Dorcolæmus sp.; B : Chrysops longicornis Macquart; C : Tabanocella stimulans Austen; D : détail du manehon de $T$. stimulans. (a, b, c : manchons des canaux des spermathèques ; b': spermathèques; b" : détails des manchons de B ; (1) : échelle de A, B, C; (2) : échelle de D et b").

sur la face dorsale de la poche copulatrice. D’après sa situation topographique, nous pouvons l'assimiler à la furca connue chez d'autres insectes. Sa forme générale est celle d'une lyre avec une base plus ou moins étendue selon les espèces et deux bras remontant vers l'orifice génital. A l'extrémité de ces bras se trouvent les 
brosses de petites épines ou de poils courts dont parlent Patton et Cragg ; ces brosses sont parfois placées sur de petites pièces assez nettement distinctes des bras eux-mêmes. C'est entre ces brosses que se situe l'orifice génital femelle (fig. I, O). A propos de cette furca il faut signaler deux plaques, un peu moins chitinisées, se trouvant de part et d'autre de son extrémité et en continuité avec lui ; les glandes annexes, dont l'orifice s'ouvre dans la poche copulatrice, semblent reposer sur ces plaques. Du côté dorsal et entre les bras de la furca, apparaissent les trois manchons qui se trou-

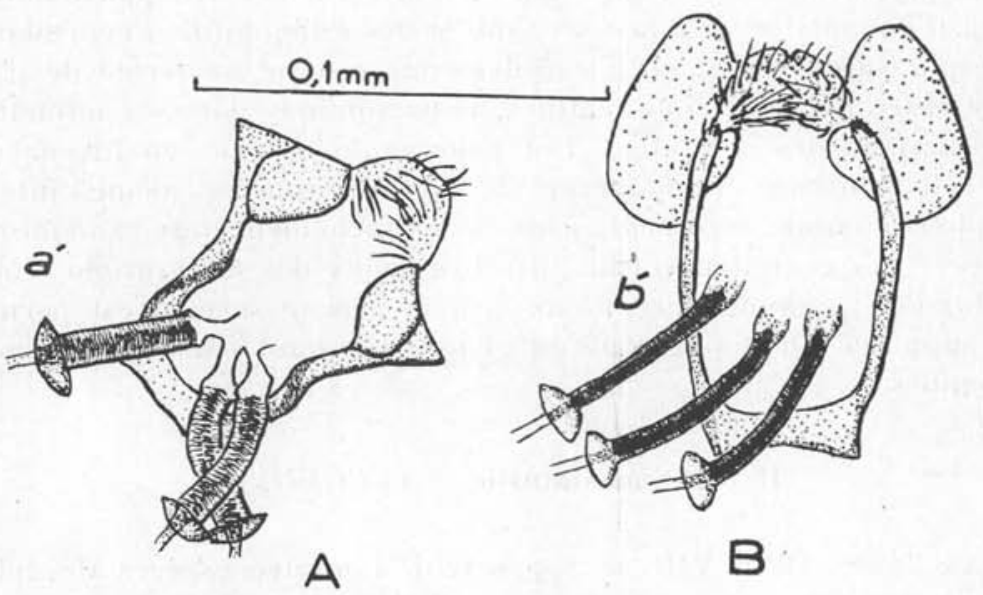

Fic. 4. - Furca chez les Tabaniinæ. A: Hippocentrum strigipenne Karsch (a' : manchons) ; B : Tabanus severinii Surcouf (b': manchons)

vent à l'origine des canaux des spermathèques (fig. II, e'). Au niveau des manchons, se termine l'oviducte (fig. I, ov). Ce sont ces manchons qui nous ont paru caractéristiques des deux sous-familles. Ainsi que le montrent les figures II, III et IV et dans l'état actuel de nos connaissances, ils sont simples chez les Pangoniinæ (genres Chrysops, Tabanocella, Dorcolaemus) alors qu'ils présentent chez les Tabaniinæe (genres Tabanus, Haematopota, Hippocentrum), à leur jonction avec le tube membraneux, une sorte de cupule lisse bien différenciée. Il est possible que la prise en considération de ce caractère remette en question la position du genre Thaumastocera Grünberg, habituellement placé dans les Tabaniinæ. Surcouf (1922) l'a considéré comme un terme de passage entre les deux sous-familles, bien que plus proche des Haematopota et l'a finalement classé dans les Tabaniinz. E. Brumpt, dans un dessin de son Précis de Parasitologie le range dans les Pangoniinæ. Pour Séguy (1946) 
c'est un Tabaniinæx. A Londres le $\mathrm{D}^{r}$ Oldroyd a confié à l'un de nous qu'il n'était pas certain que l'inclusion de ce genre dans la sousfamille des Tabaniinæe soit justifiée et, comme nous lui avions fait part de nos observations concernant le caractère en question, nous a proposé de disséquer un exemplaire de Thaumastocera sp. Cette dissection nous a révélé alors la présence des manchons caractéristiques des Pangoniinæx et d'une furca dont la forme se rapprochait de celui des Chrysops, bien que les spermathèques soient très différentes de celles, très typiques, de ce genre. Il est probablement prématuré de conclure sur ce seul caractère à l'appartenance du g. Thaumastocera à la sous-famille des Pangoniinæ ; cependant, puisque Surcouf (loc, cit.) le considérait comme un terme de passage entre les deux sous-familles, nous sommes portés à admettre que l'aspect des genitalia $\&$ fait pencher la balance en faveur du rattachement aux Pangoniinæ. Il est vrai que ce même auteur emploie comme argument pour le rattachement aux Tabaniinæ l'aspect des genitalia, voisins, dit-il, de ceux des Chrysozona (Haematopota); comme Surcouf ne spécifie pas le sexe, il est permis de supposer qu'il s'agissait de genitalia ơ que nous n'avons pas examinés.

\section{II. - Sous-famille PANGONIINAE}

Les figures V à VIII se rapportent à quatre espèces de cette sous-famille appartenant aux genres Tabanocella et Chrysops (sousgenre Kleineana).

\section{I. - Genre Tabanocella : Tabanocella stimulans Austen}

Chez cette espèce, la plaque ventraIe (fig. V, A) a, dans l'ensemble, l'aspect d'un rectangle avec deux expansions latérales prononcées, ce qui la rapproche de la forme hexagonale fréquente chez les Tabanus; mais elle ne présente ni les renforcements latéraux, ni les stries transversales des Tabaniina que nous avons vus. Les cerques (fig. V, B) sont nettement pentagonaux, avec une échancrure peu marquée à l'apex. La furca (fig. V, C) est ovalaire, sans angles nets en dehors des appendices supportant les glandes annexes; sa base est assez étendue et représente près du tiers de la longueur; moins chitinisée au centre, elle semble entourée par les bras. Les brosses de l'orifice génital (fig. V, $e$ et E) sont portées sur des pièces triangulaires nettement individualisées à l'extrémité des branches du sclérite que nous venons de décrire; elles sont formées de 6 poils relativement longs et forts, disposés en une seule rangée. Les manchons des spermathèques (fig. V, $c^{\prime}$ ) sont simples, non. dilatés à leur union avec le tube membraneux ; celui-ci, de largeur uni- 

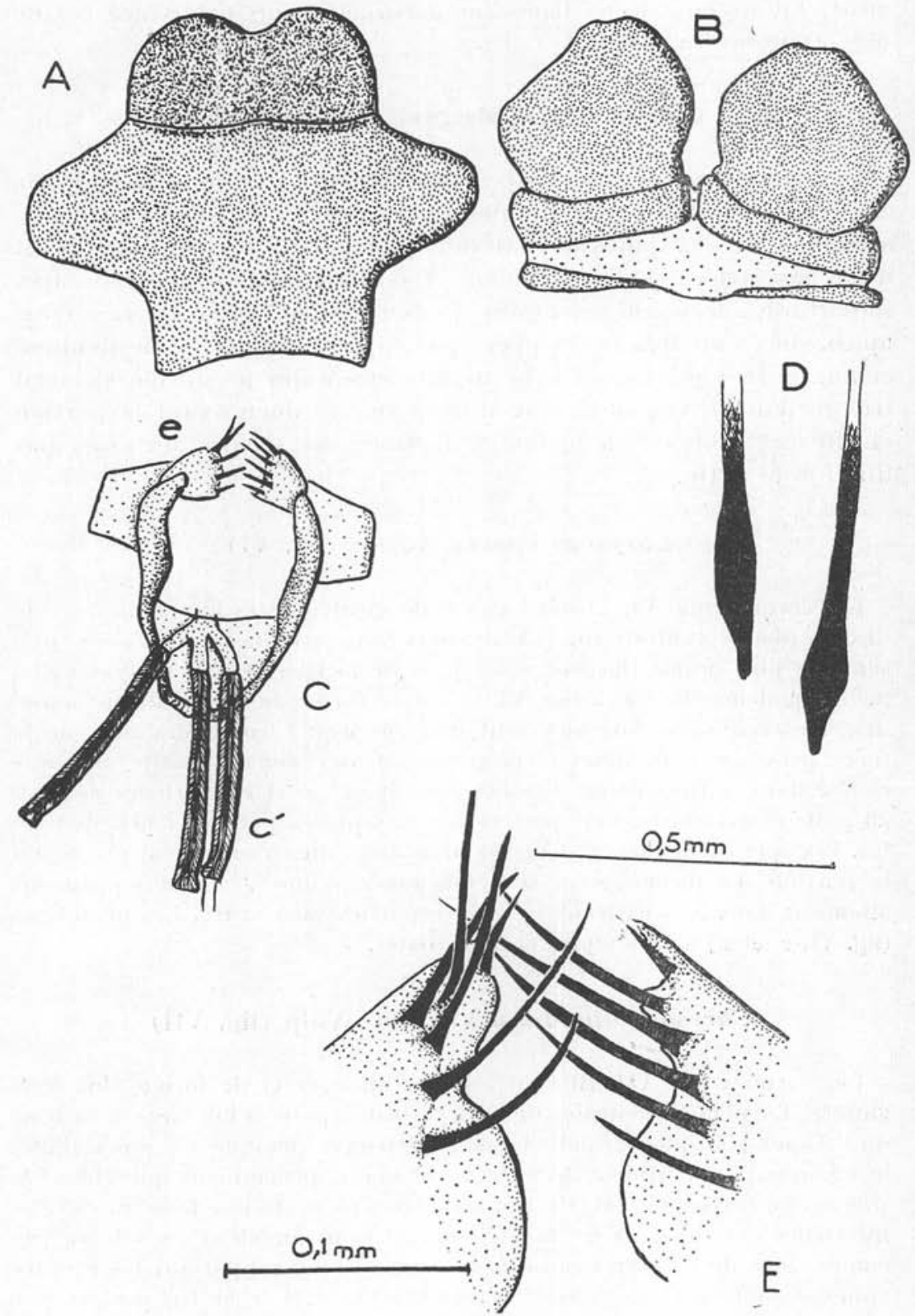

Fig. 5. - Tabanocella stimulans Aust. A : plaque ventrale; B : cerques; C : furca ( $c^{\prime}$ : manchons ; $e$ : orifice) ; D : spermathèques; E : brosses. 
forme sur presque toute sa longueur, se termine par des poches fortement chitinisées, élargies dans leur partie moyenne, tandis que l'extrémité aveugle est effilée (fig. V, D).

\section{2. - Genre Chrysops Meigen, sous-genre Kleineana}

Les trois espèces que nous avons disséquées ont beaucoup de caractères communs. La p̉laque ventrale présente des angles très arrondis, sans expansions latérales, ni zones plus chitinisées et n'est pas striée transversalement. La furca montre une base très importante, occupant au moins un tiers de sa longueur. Les spermathèques sont très développées par rapport à la taille de l'animal et aux autres pièces; le tube membraneux qui les forme s'élargit très fortement, triplant environ de diamètre, bien avant la portion chitinisée ; celle-ci, en forme de flamme, est séparée du reste par une limite nette.

Chrysops silacea Austen (fig. VI)

Les cerques (fig. VI, A) ont l'aspect de quadrilatères aux angles arrondis. La plaque ventrale (fig. VI, B), plus large à la base qu'à l'apex, présente sa plus grande largeur assez près de la base. Elle n'est pas nettement anguleuse. La furca (fig. VI, C) est en forme de lyre à angles arrondis, ses expansions latérales sont triangulaires. Chacun des bras de la furca présente deux zones d'élargissement, une dans la partie moyenne et une dans le tiers distal. Les brosses (fig. VI, $e$ et E), formées de 19 à 20 poils assez courts, sont portées par des pièces petites et peu distinctes. Les spermathèques (fig. VI, D) présentent un étranglement peu avant la portion membraneuse et sont marquées d'une série d'impressions allongées dans le sens transversal et réparties sans ordre. Les manchons (fig. VI, $c^{\prime}$ et F) sont simples et non dilatés.

\section{Chrysops dimidiata Van der Wulp (fig. VII)}

Les cerques (fig. VII, B) sont ici plus allongés et de forme plus irrégulière. La plaque ventrale (fig. VII, A) est à peine plus large à la base qu'à l'apex; sa plus grande largeur se trouve presque en son milieu; le triangle plus chitinisé de la base est moins proéminent que chez $C h$. silacea. La furca (fig. VII, C), plus allongée, présente une base aux angles inférieurs nets et dont les bords externes sont anguleux; ses bras, par contre, sont de largeur régulière. Les expansions supportant les glandes annexes sont rectangulaires. Les brosses (fig. VII, $e$ et E), portées par des pièces nettement visibles, sont formées de treize petites épines courtes et fines, groupées en deux rangs, sauf les trois dernières, qui forment un triangle. Le manchon chitineux (fig. VII, $c^{\prime}$ et F) est un peu 
élargi à son union avec le tube membraneux. Les spermathèques (fig. VII, D), de forme très voisine de celle de $C$. silacea, portent des impressions punctiformes.
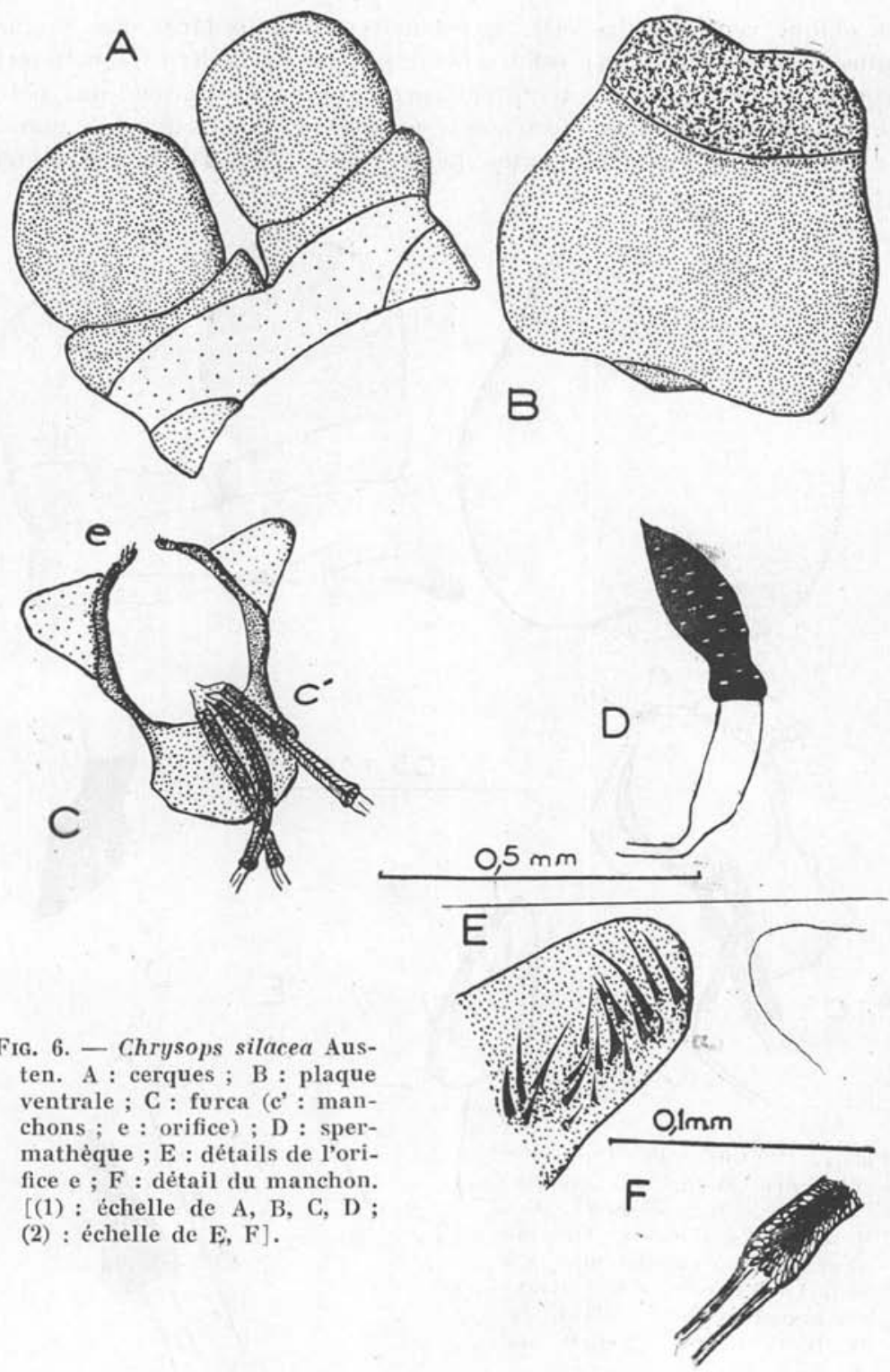

Fig. 6. - Chrysops silacea Austen. A : cerques; $B$ : plaque ventrale ; $\mathrm{C}$ : furca (c' : manchons ; e : orifice) ; D : spermathèque ; $\mathrm{E}$ : détails de l'orifice e ; F : détail du manchon. [(1): échelle de A, B, C, D ; (2) : échelle de E, F]. 


\section{Chrysops longicornis Macquart (fig. VIII)}

La plaque ventrale (fig. VIII, A) est nettement plus large que longue ; sa plus grande largeur est relativement proche de la base et nettement marquée ; la base, presque rectiligne, présente, en son milieu, une petite saillié anguleuse, mais pas de triangle particulièrement chitinisé, comme chez les deux espèces précédentes. Les cerques (fig. VIII, B) ressemblent
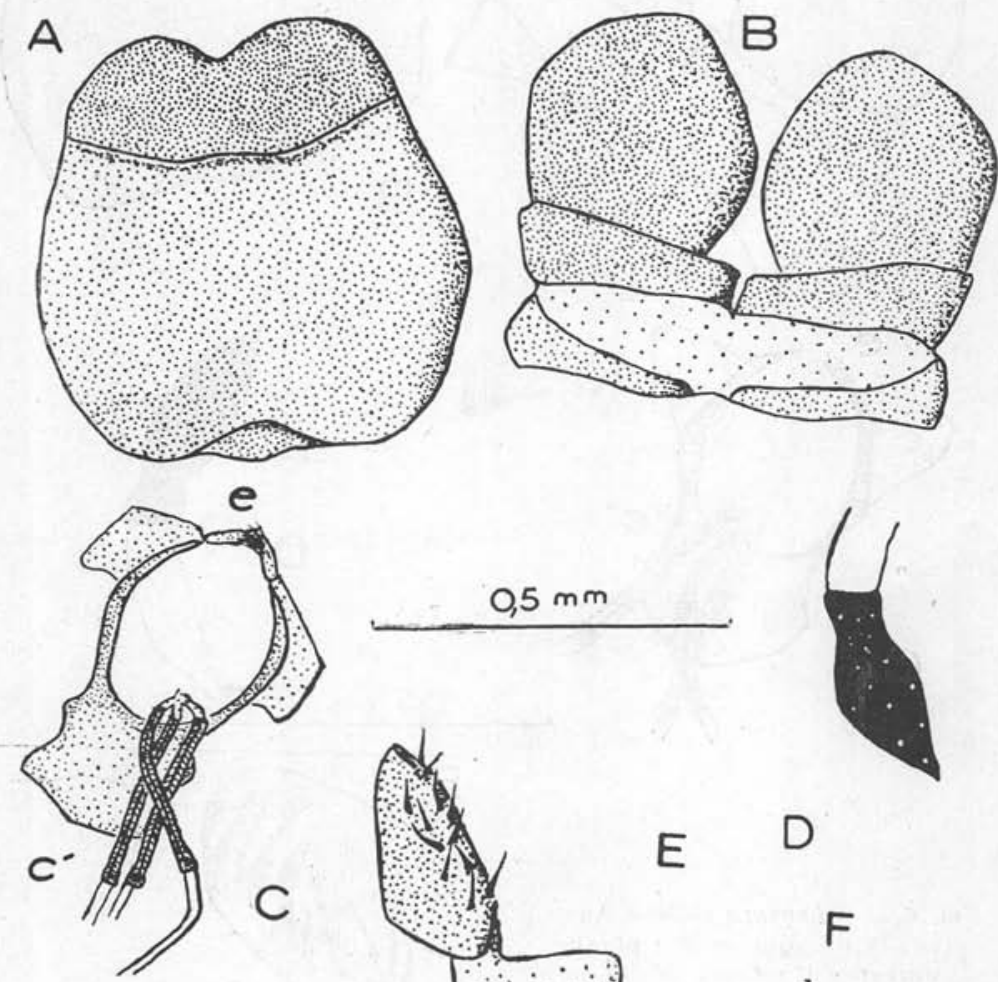

FIG. 7. - Chrysops dimidiata Van der Wulp. A : plaque ventrale; $B$ : cerques ; $C$ : furca (c': manclions; $\mathrm{e}$ : orifice); $\mathrm{D}$ : spermathèques ; $\mathrm{E}$ : détail de l'orifice; F : détail du manchon. [(1) : échelle de A, B, C, D ; (2) : éehelle de E, F].
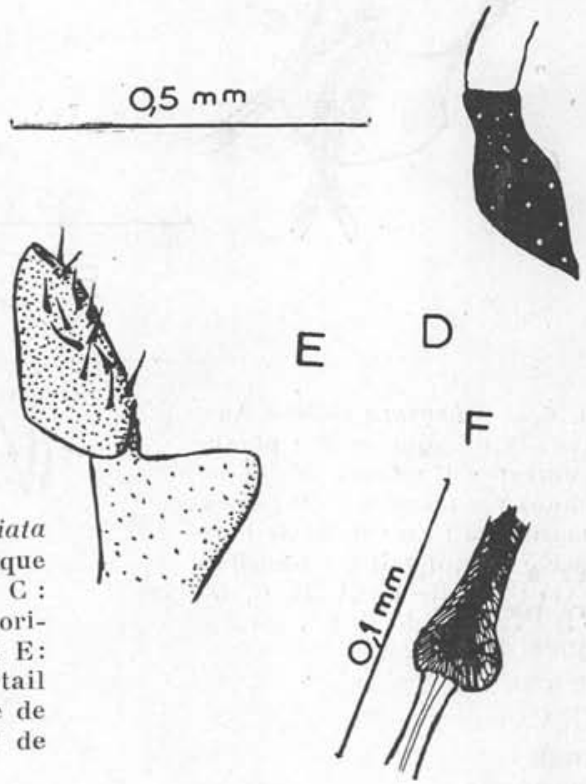

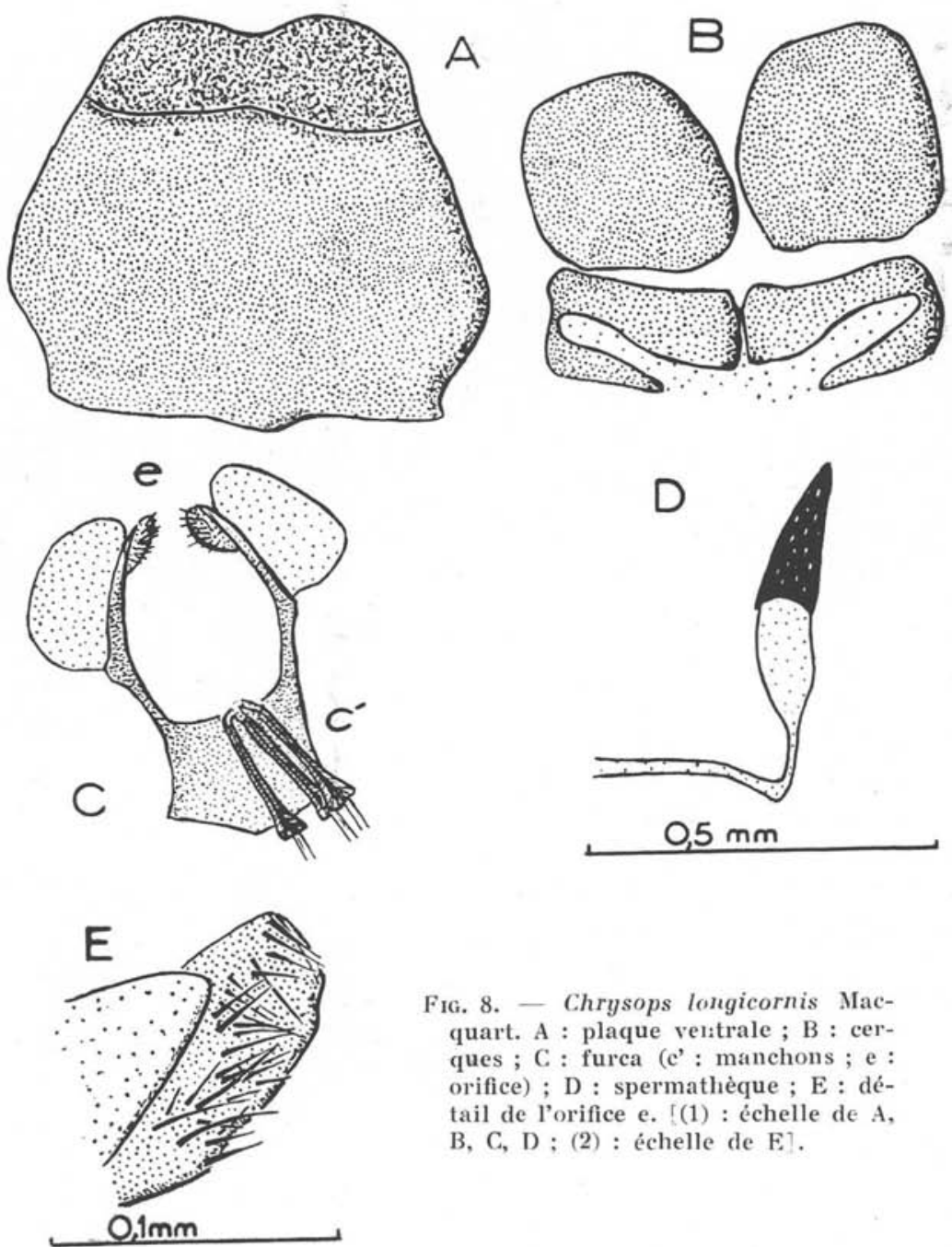

FIG. 8. - Chrysops longicornis Macquart. A : plaque velitrale ; $B$ : cerques ; C : furca ( $e^{\prime}$ : manchons ; e : orifice) ; D : spermathèque ; $\mathrm{E}$ : détail de l'orifice e. I(1) : échelle de A, $\mathrm{B}, \mathrm{C}, \mathrm{D}$ : (2) : échelle de E?.

assez à ceux de $C$. silacea, mais, ainsi que nous l'avons dit plus haut, leurs pièces basales ne paraissent formées que de deux sclérites symétriques au lieu de quatre en raison de l'union étroite de ces pièces du côté externe ; leur forme contournée est caractéristique. La furca (fig. VIII, C) a, chez cette espèce, comme chez les deux précédentes, une base à angles nets, mais cette base ne présente aucun élargissement latéral ; les bras de la furca sont au contraire élargis à peu près à la moitié de 
leur longueur; les deux expansions des glandes annexes sont allongées et plutôt ovalaires. Les brosses de l'orifice génital (fig. VIII, $e$ et E) sont portées par de petites pièces nettement visibles, situées à l'intérieur des bras et non à leur extrémité ; elles se composent d'environ 25 poils assez courts et très fins, disposés sans ordre apparent. Les manchons chitineux (fig. VIII, $c^{\prime}$ ) sont très nettement élargis avant leur jonction avec le canal membraneux. Les spermathèques (fig. VIII, D) ne portent pas de rétrécissement dans leur partie chitineuse et sont ornées d'impressions allongées dans le sens de la longueur.

\section{BIBLIOGRAPHIE}

Brygoo (E. R. R.). - Tabanides d'Ayos. Bull. Soc. Etudes Camer., 1950.

Collin (J. E.). - A note on certain structural distinctions between females of Therioplectes tropicus L., solstitialis $\mathrm{Mg}$. and distinguendus Verr. The Entom. Month. Mag., 76, 1940, p. 78-79.

Oldroyd (H.). - The Horse-flies (Dipt. Tabanidæ) of the Ethiopian region, vol. I, Hamatopota and Hippocentrum. Publ. British Museum (Nat. Hist.), 1952.

Patron (W. E.) et Cragig (F. W.). - A textbook of Medical Entomology, 1913.

SÉGUY (E.). - Tabanides nouveaux et peu connus d'Afrique. Tabanus des groupes maculatissimus et latipes; deseription d'un nouveau Thaumastocera. Encycl. Entom., Diptera, sér. B, 2, 1946, n 10, 22-26.

- La biologie des Diptères, P. Lechevallier édit., Paris, 1950.

- Diptères ; généralités in P.-P. Grassé : Traité de Zoologie Générale, Masson édit., Paris, 1951.

Surcouf (J.-M.-R.). - Diptères nouveaux ou peu connus. Ann. Soc. Entom. France, 91, 1922, (5), p. 237-244.

(Institut Pasteur de Brazzaville, Institut d'Etudes centrafricaines, Office de la Recherche Scientifique et Technique d'Ontre-Mer) 